\title{
Optical Stark Decelerator for Molecules
}

\author{
R. Fulton, A. I. Bishop, and P. F. Barker \\ Department of Physics, School of Engineering and Physical Sciences, Heriot-Watt University, \\ Edinburgh, EH14 4AS, United Kingdom
}

(Received 1 September 2004; published 9 December 2004)

\begin{abstract}
We demonstrate a single stage optical Stark decelerator for neutral molecules which is capable of reducing the translational energy of benzene molecules within a molecular beam by $15 \%$ in a single pulsed Gaussian optical field of $15 \mathrm{~ns}$ duration. The change in velocity induced by the optical potential is measured by the change in the time of flight of benzene molecules ionized by a second optical field after the slowing field is turned off. A maximum reduction in the velocity of $25 \mathrm{~m} / \mathrm{s}$ was measured using a single Gaussian beam with a peak intensity of $1.6 \times 10^{12} \mathrm{~W} / \mathrm{cm}^{2}$ corresponding to a maximum well depth of $253 \mathrm{~K}$.
\end{abstract}

DOI: 10.1103/PhysRevLett.93.243004

PACS numbers: 33.80.Ps, 32.80.Lg, 39.10.+j

Slow cold molecules have important applications in cold molecular collisions and chemistry [1,2], and are potential new frequency standards. Cold trapped polar molecules are of interest for studying molecular dipoledipole interactions, and a quantum computation scheme based on cold trapped polar molecules has been proposed [3]. Heavy cold polar molecules are currently seen as ideal candidates to test theories beyond the standard model in the search for a measurable electric dipole moment of the electron [4]. The large de Broglie wavelengths of massive cold molecules and nanoparticles are currently of interest for molecular matter-wave interferometry, offering a new tool for exploring the boundary between the classical and quantum motion of larger molecules and nanoparticles [5,6].

The creation of cold molecules using the techniques developed for atomic species cannot readily be applied to molecules, because of the lack of a closed set of cycling transitions, due primarily to the absence of strict selection rules between vibrational levels. Cold molecules can be created by collisions induced by photoassociation [7] and on Feshbach resonances [8], but these processes are limited to the subset of atomic species which can be laser cooled. A number of other more general approaches to cold molecule creation include buffer gas cooling [9] and electrostatic Stark deceleration of cold polar molecules produced in a free jet $[10,11]$. Cold slow molecules have also been created by a rotating jet [12] and by collisions with atoms in crossed molecular beams [13]. In buffer gas cooling, a molecular or atomic species is cooled within a cryogenic cell containing $\mathrm{He}^{3}$ and $\mathrm{He}^{4}$ gas where they are thermalized by collisions with the cold helium. Temperatures of $400 \mathrm{mK}$ have been achieved with a number of magnetically trapped molecular species [9]. Stark deceleration of polar molecules is produced when a weak or a strong field seeking state loses energy as it moves into a nonuniform electrostatic field $[10,11,14,15]$.

In analogy with the Stark deceleration of polar molecules in electrostatic fields, the second order Stark shift within high intensity optical fields offers another even more general route to the deceleration of cold molecules. Recently, quasielectrostatic potentials, created by faroff-resonant pulsed optical fields in the $10^{12} \mathrm{~W} / \mathrm{cm}^{2}$ range, have been used to manipulate molecules in molecular beams and deflection, separation, and rotation of a range of species have been demonstrated [16-18]. This type of molecular manipulation relies on the polarizability interaction between the molecule and the optical field. As all atoms, molecules, and clusters are polarizable to some extent, it appears to be a very general method that is suitable for many species which cannot be manipulated using conventional cooling and trapping techniques [19]. The creation of cold, slow molecules by deceleration, via quasielectrostatic potentials, has been proposed [16,19-21].

In this Letter we describe an optical decelerator utilizing the optical dipole force induced by pulsed fields in the $10^{12} \mathrm{~W} / \mathrm{cm}^{2}$ range. Using this scheme we have decelerated cold benzene molecules in a supersonic jet and measured a reduction in energy of the molecules by up to $15 \%$ in a single stage. The potential energy of a molecule in a far-off-resonant optical field is given by $U(r, t)=$ $-\frac{\alpha}{2 \varepsilon_{0} c} I(r, t)$, where $I(r, t)$ is the intensity of the field and $\alpha$ is the effective polarizability of the molecule. Using fields tuned far below all electronic transitions implies that all neutral particles are high field seeking [22], and, additionally, very little heating should result due to the application of far-off-resonant and short pulse optical fields (15 ns) [23]. We use a single focused laser beam with a Gaussian intensity profile $I(x, y, t)=$ $I_{0} A(t) e^{-2\left[\left(x^{2}+y^{2}\right) / \omega_{0}^{2}\right]}$, where $A(t)$ is a smooth time dependent modulation, typical of $Q$-switched lasers. This potential has a maximum well depth of $253 \mathrm{~K}$ for benzene molecules with a peak intensity of $1.6 \times 10^{12} \mathrm{~W} / \mathrm{cm}^{2}$ and an average polarizability of $11.6 \times 10^{-40} \mathrm{~cm}^{2} / \mathrm{V}$. The large magnitude of this potential is six orders greater than is commonly used in cw quasielectrostatic traps 
employed in atom optics. This large potential is required to decelerate molecules that are traveling at supersonic speeds of hundreds of meters per second, over the short (15 ns) duration of the pulsed field. The magnitude of the dipole force on a molecule is given by the gradient of the optical potential. We need consider only the gradient in the radial direction within the $2 \mathrm{~mm}$ Rayleigh range of the focused beam where there is no significant intensity gradient along the beam axis. The orientation of the molecular beam to the focused Gaussian optical beam used in our slowing experiments is shown in Fig. 1.

Molecules within the field experience forces both perpendicular and parallel to the beam direction. The force perpendicular to the molecular beam in the $x$ direction shown in Fig. 1 results in focusing or deflection, which has been studied for a number of molecules [16-18]. The force along the molecular beam in the $y$ direction can result in both deceleration and acceleration of the beam. The motion of molecules along the center of the potential in the $y$ direction is well described by the phase space $(y, v)$ plot of Fig. 2. The equations of motion for molecules along the $y$ axis are given by $\frac{d v}{d t}=-\frac{2 \alpha I y}{m \varepsilon_{0} c \omega_{0}^{2}} ; \frac{d y}{d t}=v$. To elucidate the essential features of the motion within the optical potential, we consider a Gaussian beam of constant intensity for the duration of the pulse. The solid lines in Fig. 2 represent lines of equal energy and are the trajectories of molecules in the $(y, v)$ plane. The zero position corresponds to the center of the optical potential along the $y$ direction as defined in Fig. 1, and the arrows represent the direction that molecules move along these equal energy lines. The thickest closed line in the figure is the separatrix, which divides the phase space into two regions. Inside, the molecules are trapped by the potential as long as the potential is switched on, while outside they

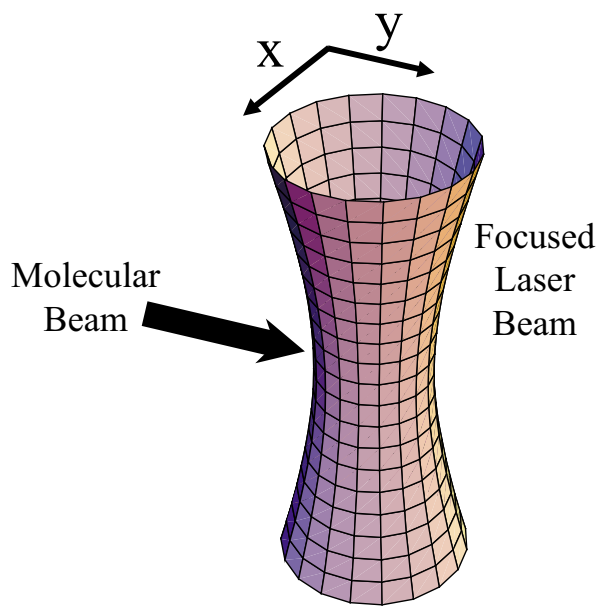

FIG. 1 (color online). A diagram illustrating the orientation of the molecular beam with respect to the optical Stark decelerating field created by a focused Gaussian beam. Slowing occurs along the $y$ axis in the direction of the molecular beam. are untrapped but their velocity is strongly perturbed by the potential. The narrow horizontal line represents the velocities and positions of molecules in a molecular beam propagating along the $y$ axis when the optical field is instantaneously switched on. These molecules have a well defined velocity, but are uniformly distributed in position along the $y$ axis. The thicker shorter curves represent the evolution in velocity and position of molecules over a $15 \mathrm{~ns}$ period which started within the molecular beam at different positions along the $y$ axis. Molecules that are upstream of the optical potential (laser focus) corresponding to $y=0$ are accelerated, while those downstream are decelerated. In this diagram the maximum change in velocity is $46 \mathrm{~m} / \mathrm{s}$ even though molecules move only a short distance of $4.5 \mu \mathrm{m}$ along the $y$ axis. For the short pulse durations that we consider, both trapped and untrapped motion leads to deceleration if the molecules are downstream of the laser focus at $y>0$.

To study the deceleration of cold benzene molecules in the Gaussian optical potential we produce a cold supersonic beam by seeding benzene into a xenon supersonic expansion created by a pulsed solenoid valve. The $\mathrm{Xe} / \mathrm{C}_{6} \mathrm{H}_{6}$ mixture was expanded into a vacuum chamber, initially at $10^{-7}$ torr, through an orifice of diameter $500 \mu \mathrm{m}$. A collimated $\mathrm{Xe} / \mathrm{C}_{6} \mathrm{H}_{6}$ molecular beam at $2.3 \mathrm{~K}$ was formed in a second differentially pumped chamber $\left(10^{-7}\right.$ torr $)$ by allowing the central portion of the jet to pass through a $2 \mathrm{~mm}$ skimmer orifice. The slowing Gaussian optical potential was created by focusing the output of an infrared (IR) injection seeded $Q$-switched Nd-doped yttrium aluminum garnet (Nd:YAG) laser $(\lambda=1064 \mathrm{~nm})$ to a $e^{-2}$ radius of $20 \mu \mathrm{m}$. The laser beam was linearly polarized orthogonal to the molecular beam direction and the injection

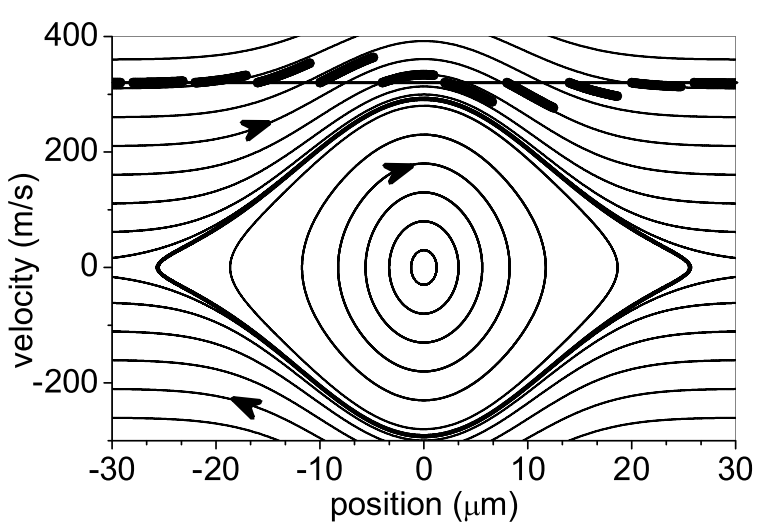

FIG. 2. A phase space plot showing the motion of trapped and untrapped particles within a Stark decelerating field for a pulsed laser field with a constant intensity for the duration of the laser pulse. The solid lines represent lines of equal energy, and the arrows represent the direction that molecules move along these lines. The thickest closed line is the separatrix which encloses the trajectories of the trapped molecules. 
seeded laser produced a temporally smooth intensity profile with a full width half maximum pulse width of $15 \mathrm{~ns}$ and a peak intensity at the focus of $1.6 \times$ $10^{12} \mathrm{~W} / \mathrm{cm}^{2}$. This temporally smooth profile was required to prevent the loss of neutral molecules through ionization and dissociation from the high intensity spikes created by longitudinal mode beating when the laser was unseeded. When the optical field is applied for the duration of the laser pulse (15 ns), the molecules traveling at supersonic speeds $(320 \mathrm{~m} / \mathrm{s})$ do not travel completely through the optical potential $(4.5 \mu \mathrm{m})$ during the pulse, and therefore only those species that are located within the focus receive an impulsive kick from the potential. To detect and measure the velocity of the molecules they were ionized using a $1+1$ resonantly enhanced multiphoton ionization (REMPI) scheme. The first step was resonant with the ${ }_{0}^{1} 6 S_{1} \leftarrow S_{0}$ transition of benzene in the $\mathrm{UV}$ at a wavelength of $259 \mathrm{~nm}$ [24]. We estimate velocity changes of up to $2.4 \mathrm{~m} / \mathrm{s}$ could be induced by recoil of the ions in our REMPI scheme. The velocity of the benzene molecules was measured $60 \mathrm{~ns}$ after the IR field had been turned off to prevent perturbation of the benzene ion velocity induced by the IR field. The spatial resolution of the UV probe beam was estimated to be approximately $2 \mu \mathrm{m}$. Using measurements of pulse energy, pulse duration, and spot size we estimate a maximum intensity of this beam at its focus to be $10^{7} \mathrm{~W} / \mathrm{cm}^{2}$. At this intensity the velocity of the molecules is not significantly perturbed by the dipole force induced by this field. The velocity of the neutral benzene molecules slowed by the IR field was measured by the time of flight of the benzene ions within a Wiley-McLaren type time-of-flight (TOF) mass spectrometer. As the focus of the IR laser beam was uniform only within the Rayleigh range of approximately $2 \mathrm{~mm}$, a $150 \mu \mathrm{m}$ slit was placed on the extractor electrode to limit the detection to those ions created within the laser focus. The velocity of molecules at different positions along the $y$ axis was measured as a function of distance along the molecular beam direction by scanning the IR beam along the $y$ axis with a resolution of $1 \mu \mathrm{m}$. Figure 3 contains a typical time-of-flight deceleration profile recorded $27 \mu \mathrm{m}$ downstream from the IR focus. The change in TOF was converted to velocity from calculations of the velocity-TOF dispersion relation determined by numerical simulation of the motion of xenon ions within the spectrometer, in good agreement with measurements made in the absence of the decelerating field. The electrode voltages of the spectrometer were selected to produce a position focusing condition where ions of the same mass and velocity arrive at the same time on the multichannel plate detector irrespective of their position within the IR field. However, there will be some broadening in the averaged TOF signal due to shot to shot fluctuations in the position $(5 \mu \mathrm{m})$ and timing $(1 \mathrm{~ns})$ of the IR and UV beams. The measured velocity shift, as

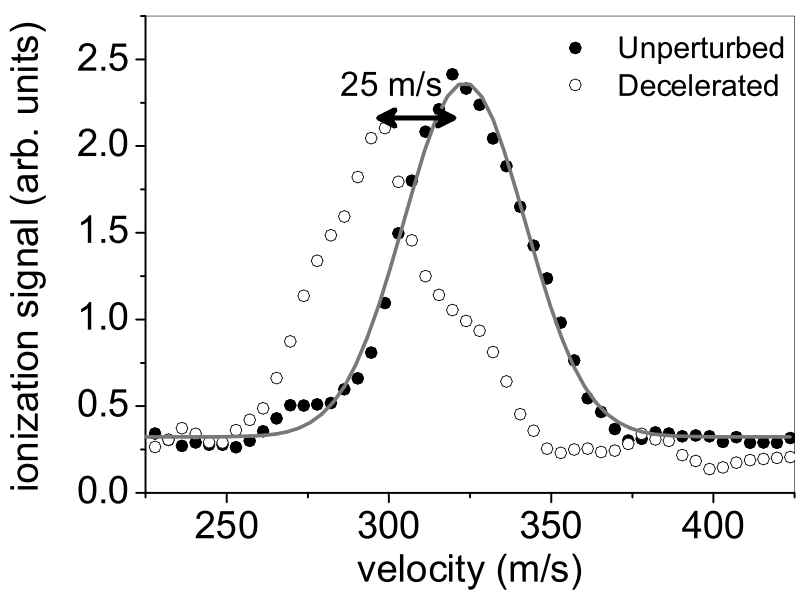

FIG. 3. A typical velocity profile of benzene molecules slowed in the decelerator derived from a time-of-flight measurement. For comparison, the velocity profile of unperturbed molecules at a temperature of $2.4 \mathrm{~K}$ is also shown illustrating a decrease in velocity of $25 \mathrm{~m} / \mathrm{s}$ following deceleration and an average deceleration of $10^{8} \mathrm{~g}$. The solid line is a Gaussian fit to the unperturbed distribution which gives a temperature of $2.3 \mathrm{~K}$.

determined from the center of the distribution, will be unaffected by both of these fluctuations. Also shown is a profile recorded for the unperturbed molecular beam when no IR field was present. As the TOF is linearly proportional to molecular velocity in the $y$ direction, each profile represents the velocity distribution of molecules in this direction. A fit to the unperturbed TOF profile shown in Fig. 3 indicates a translational temperature of $2.3 \mathrm{~K}$. The velocity profile induced by the dipole

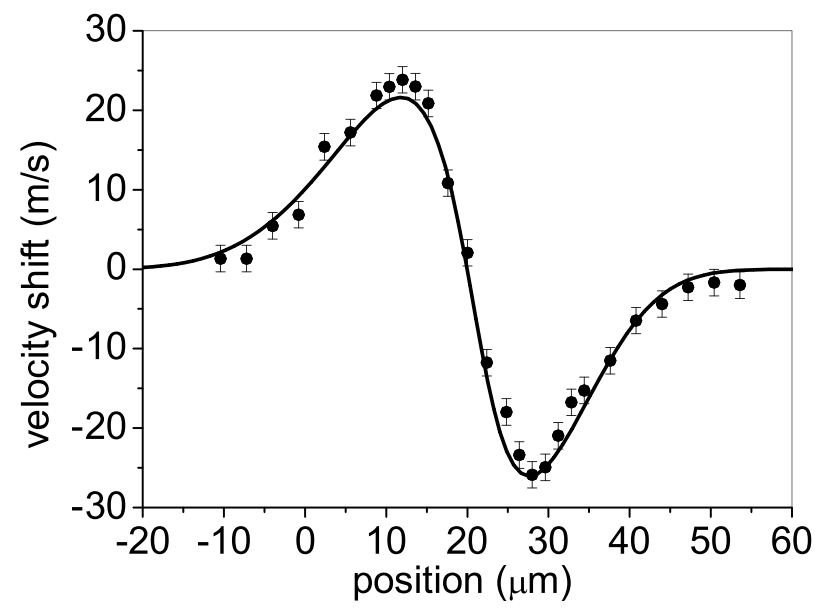

FIG. 4. Velocity changes induced by the optical potential along the molecular beam direction ( $y$ axis in Fig. 1). The points are experimental data and the line was calculated from measurements of the beam waist, energy, and the temporal profile. The focused laser beam had a peak intensity of $1.6 \times$ $10^{12} \mathrm{~W} / \mathrm{cm}^{2}$. 
force along the $y$ axis is shown in Fig. 4. Each point was determined by measuring the change in the velocity of the center of the velocity distribution between the decelerated and unpertubed distribution. These results show that benzene was slowed by up to $25 \mathrm{~m} / \mathrm{s}$ in a single pulse and that slightly larger changes in velocity can be achieved for deceleration when compared to acceleration. This occurs because molecules that are slowed spend more time at a lower velocity where they are more strongly perturbed by the potential. The velocity changes due to deceleration and acceleration are not symmetric either side of the central point because the faster molecules spread out more rapidly than the slower molecules over the $60 \mathrm{~ns}$ period between application of the IR slowing field and the UV probe. Figure 4 is the velocity profile calculated by integration of the equations of motion using our measurements of pulse shape, beam energy, and spot size. The agreement is very good given that our measurement of intensity has a $20 \%$ uncertainty. The figure also shows that deceleration and acceleration can be achieved with each process dependent on the initial position of the molecules with respect to the center of the IR field.

We have produced a single stage optical Stark decelerator for benzene molecules demonstrating a maximum reduction in translation energy of $15 \%$ in a single laser pulse and an average deceleration of $10^{8} \mathrm{~g}$. This technique should be applicable to many atoms, molecules, and clusters, which cannot be efficiently slowed using other methods. More recently we have used the same fields to manipulate the motion of the Xe carrier gas [25]. Our calculations indicate that an additional identical stage, or a single stage with longer pulse duration of $120 \mathrm{~ns}$ duration with the same intensity, would decelerate the molecules in the jet to zero velocity. We estimate that benzene at a density of $10^{15} \mathrm{~cm}^{-3}\left(10^{9}\right.$ molecules $)$ could be slowed in a single laser pulse at a distance of 20 nozzle diameters from the jet. If a far-off-resonant trap [23] with a well depth of $1 \mathrm{mK}$ was overlapped with the slowed distribution, approximately $10^{7}$ molecules could be trapped. This estimate is an upper bound, limited by collisions with the xenon carrier gas during deceleration, while the trap lifetime would be limited to less than a second by the background pressure after deceleration. This density of cold molecules would already be useful for many applications, and as a starting point for further cooling schemes such as cavity Doppler cooling [26,27]. Finally, we are currently investigating the effects of molecular alignment in our experiments and expect to enhance the slowing by up to a factor of 2 for benzene [28].

This work was supported by the U.K.'s Engineering and Physical Sciences Research Council under Grant No. GR/R53104/01.

[1] N. Balakrishnan and A. Dalgarno, Chem. Phys. Lett. 341, 652 (2001).

[2] K. Burnett et al., Nature (London) 416, 225 (2002).

[3] D. DeMille, Phys. Rev. Lett. 88, 067901 (2002).

[4] J. J. Hudson et al., Phys. Rev. Lett. 89, 023003 (2002).

[5] L. Hackermúller et al., Phys. Rev. Lett. 91, 090408 (2003).

[6] B. Brezger et al., Phys. Rev. Lett. 88, 100404 (2002).

[7] U. Schlöter, C. Silber, and C. Zimmermann, Appl. Phys. B 73, 801 (2001).

[8] C. A. Regal et al., Nature (London) 424, 47 (2003).

[9] J. D. Weinstein et al., Nature (London) 395, 148 (1998).

[10] H. L. Bethlem et al., Nature (London) 406, 491 (2000).

[11] H. L. Bethlem et al., Phys. Rev. Lett. 88, 133003 (2002).

[12] M. Gupta and D. Herschbach, J. Phys. Chem. A 103, 10670 (1999).

[13] M. S. Elioff, J. J. Valentini, and D.W. Chandler, Science 302, 1940 (2003).

[14] J. R. Bochinski et al., Phys. Rev. Lett. 91, 243001 (2003).

[15] M. R. Tarbutt et al., Phys. Rev. Lett. 92, 173002 (2004).

[16] H. Stapelfeldt et al., Phys. Rev. Lett. 79, 2787 (1997); H. Sakai et al., Phys. Rev. A 57, 2794 (1998).

[17] D. M. Villeneuve et al., Phys. Rev. Lett. 85, 542 (2000).

[18] B. S. Zhao et al., Phys. Rev. Lett. 85, 2705 (2000); H. S. Chung et al., J. Chem. Phys. 114, 8293 (2001).

[19] B. Friedrich, Phys. Rev. A 61, 025403 (2000).

[20] P. F. Barker and M. N. Shneider, Phys. Rev. A 66, 065402 (2002).

[21] G. Dong, W. Lu, and P. F. Barker, Phys. Rev. A 69, 013409 (2004).

[22] T. Seideman, J. Chem. Phys. 111, 4397 (1999).

[23] T. Takekoshi, J. R. Yeh, and R. J. Knize, Opt. Commun. 114, 421 (1995).

[24] M. Okruss, R. Muller, and A. Hese, J. Mol. Spectrosc. 193, 293 (1999).

[25] P. Barker, R. Fulton, and A. I. Bishop (to be published).

[26] P. Domokos and H. Ritsch, J. Opt. Soc. Am. B 20, 1098 (2003).

[27] A. T. Black, H.W. Chan, and V. Vuletić, Phys. Rev. Lett. 91, 203001 (2003).

[28] H. Stapelfeldt and T. Seideman, Rev. Mod. Phys. 75, 543 (2003). 